PSFC/JA-97-23

\title{
ELMs and Fast Edge Fluctuations in Alcator C-Mod
}

\author{
J.A. Snipes, R.S. Granetz, M. Greenwald, A.E. Hubbard, \\ J. Irby, J. Kesner, S. Migliuolo, T.S. Pedersen, \\ J. Ramos, J. Rice, P.C. Stek ${ }^{1}$, Y. Takase ${ }^{2}$, S.M. Wolfe
}

September 1997
Plasma Science and Fusion Center Massachusetts Institute of Technology
Cambridge, MA 02139

\footnotetext{
${ }^{1}$ Present address JET Propulsion Laboratory, Pasadena, CA, USA.

${ }^{2}$ Present address Dept. of Physics, University of Tokyo, Tokyo, Japan.
}

Submitted to Plasma Physics and Controlled Fusion.

This work was supported in part by the U. S. Department of Energy Contract No. DEAC02-78ET51013. Reproduction, translation, publication, use and disposal, in whole or in part by or for the United States government is permitted. 


\title{
ELMs and fast edge fluctuations in Alcator C-Mod*
}

\author{
J A Snipes ${ }^{\dagger}, \mathbf{R}$ S Granetz ${ }^{\dagger}, \mathbf{M}$ Greenwald $^{\dagger}$, A E Hubbard ${ }^{\dagger}$, \\ I $\mathbf{H}$ Hutchinson ${ }^{\dagger}, \mathbf{J}$ Irby $^{\dagger}, \mathbf{J}$ Kesner ${ }^{\dagger}, \mathbf{S}$ Migliuolo $^{\dagger}$, \\ T S Pedersen ${ }^{\dagger}, \mathbf{J}$ Ramos ${ }^{\dagger}, \mathrm{J} \mathrm{Rice}^{\dagger}, \mathbf{P}$ C Stek ${ }^{\ddagger}, \mathbf{Y}$ Takase $^{\S}$ and \\ S M Wolfe ${ }^{\dagger}$
}
*Supported by U.S. D.O.E. Contract No. DE-AC02-78ET51013.
- †MIT Plasma Science and Fusion Center, Cambridge, MA USA
$\stackrel{\ddagger}{\ddagger}$ present address JET Propulsion Laboratory, Pasadena, CA USA

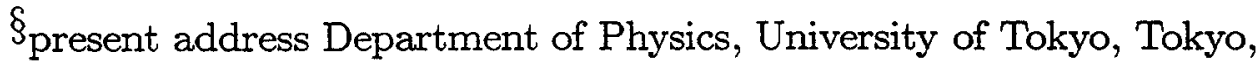 Japan

\begin{abstract}
Just above the $\mathrm{H}$-mode threshold power at low density, small amplitude high repetition rate $(1-5 \mathrm{kHz})$ Type III Edge Localized Modes (ELMs) are observed. At higher input power, the H-modes become ELM-free. At high density and high input power $\left(\bar{n}_{e}>\right.$ $2.2 \times 10^{20} \mathrm{~m}^{-3}, \mathrm{P}_{\text {in }}>2 \mathrm{MW}$ ), the plasma often enters a new H-mode regime now called the Enhanced $\mathrm{D}_{\alpha} \mathrm{H}$-mode, which is a steady-state $\mathrm{H}$ mode often without distinct ELMs but with high levels of $\mathrm{D}_{\alpha}$ emission. Enhanced $D_{\alpha} H$-modes are accompanied by persistent high frequency broadband $(50-400 \mathrm{kHz})$ density and magnetic fluctuations. Above about $\bar{n}_{e}>3 \times 10^{20} \mathrm{~m}^{-3}$, the Enhanced $\mathrm{D}_{\alpha} \mathrm{H}$-modes may also exhibit bursting or continuous low $\mathrm{m}, \mathrm{n}$ coherent magnetic fluctuations with frequencies from $60-90 \mathrm{kHz}$. In $\mathrm{H}$-mode, steep edge gradients are observed with a nine channel ECE polychromator and a 38 channel high resolution edge soft $x$ ray array. The soft $x$ ray pedestal width remains around 3 to $4 \mathrm{~mm}$ in ELM-free H-mode and between Type III ELMs. The pedestal collapses within $100 \mu$ sec during an ELM. Calculations indicate that the plasma in $\mathrm{H}$-mode is often near ideal ballooning first stability limits, but no Type I ELMs have yet been identified. Edge micro-tearing modes destabilized by a steep temperature gradient are also considered as a possible explanation of the observed fluctuations during Enhanced $\mathrm{D}_{\alpha} \mathrm{H}$-mode.
\end{abstract}

Submitted to Plasma Phys. Control. Fusion 


\section{Introduction}

Edge localized fluctuations have been identified during H-mode since the discovery of H-modes on ASDEX [1]. Previous work has classified Edge Localized Modes (ELMs) into three types [2] depending on their amplitude and frequency as well as on edge stability properties. On Alcator C-Mod [3], of these types, only Type III ELMs have been identified with no characteristic progression to Type I ELMs as the edge gradients increase, at least up to the maximum available input power (4.5 MW). The progression from Type III ELMs to ELM-free H-mode is observed as the input power increases above the threshold power to achieve $\mathrm{H}$-mode [4]. With increasing input power, the plasma can sustain increased density and remain in $\mathrm{H}$-mode. At high input power and high density $\left(\mathrm{P}_{\text {in }}>2 \mathrm{MW}, \bar{n}_{e}>2.2 \times 10^{20} \mathrm{~m}^{-3}\right.$ ), instead of entering a Type I ELMy regime, the plasma enters a new regime now called an Enhanced $D_{\alpha}$ (EDA) H-mode [5-7], which is a steady-state H-mode that usually has no clear ELMs but has a high level of $D_{\alpha}$ emission that can reach or exceed L-mode levels. Broadband high frequency $(50-400 \mathrm{kHz})$ density and magnetic fluctuations are observed during the enhanced $\mathrm{D}_{\alpha}$ phase. After several confinement times into a well developed EDA H-mode, when the density exceeds about $\bar{n}_{e}>3 \times 10^{20} \mathrm{~m}^{-3}$, bursting or continuous high frequency coherent magnetic $(60-90 \mathrm{kHz})$ fluctuations have also been observed [8]. These high frequency density and magnetic fluctuations appear to be localized near the edge of the plasma and they occur only at high density and high input power. The edge gradients under these conditions, and often also during ELM-free and Type III ELMy H-modes, are near ballooning or micro-tearing stability limits $[9,10]$.

\section{Edge Fluctuation Diagnostics}

Magnetic fluctuations in C-Mod are measured with several arrays of poloidal field pick-up coils at the inner wall and on the outboard limiters. The inner wall coils are beneath molybdenum tiles, which limit the frequency response to about $150 \mathrm{kHz}$. The outboard limiter coils are covered only by a $0.5 \mathrm{~mm}$ stainless steel cover that limits the frequency response to about $300 \mathrm{kHz}$. Presently, 48 coils channels can be sampled at rates up to $1 \mathrm{MHz}$ each with $128 \mathrm{~K}$ of samples.

Density fluctuations are measured with an $\mathrm{AM}$ reflectometer[11] at $88 \mathrm{GHz}$ operating in O-mode with a density cutoff at $9.6 \times 10^{19} \mathrm{~m}^{-3}$, which is in the steep gradient region just inside the separatrix during $\mathrm{H}$-mode. This diagnostic was designed to measure the density profile rather than fluctuations. Fluctuations in the phase are measured while fluctuations in the amplitude $\left(\tilde{n}_{e} / n_{e}\right)$ cannot be determined with the present system. The $88 \mathrm{GHz}$ channel was sampled at $1 \mathrm{MHz}$ with a bandpass filter that allows up to $400 \mathrm{kHz}$ fluctuations to be measured.

Edge electron temperature pedestals are measured with a 9 channel ECE grating polychromator, which normally has about $2 \mathrm{~cm}$ channel separation and $50 \mu \mathrm{sec}$ time resolution. The effective radial resolution can also be somewhat improved to $\sim 1 \mathrm{~cm}$ by 
ramping the magnetic field and sweeping the channels across the edge steep gradient region. A high resolution edge soft $\mathrm{x}$ ray imaging array of 38 photodiodes with a spatial resolution of about $1.7 \mathrm{~mm}$ and a time resolution of $12 \mu \mathrm{sec}$ also measures a combination of temperature and density profiles in the edge. In the absence of more detailed edge density and temperature measurements, the edge soft $\mathrm{x}$ ray pedestal widths are used as a qualitative indicator of how the edge density and temperature pedestals change with time in the $\mathrm{H}$-mode.
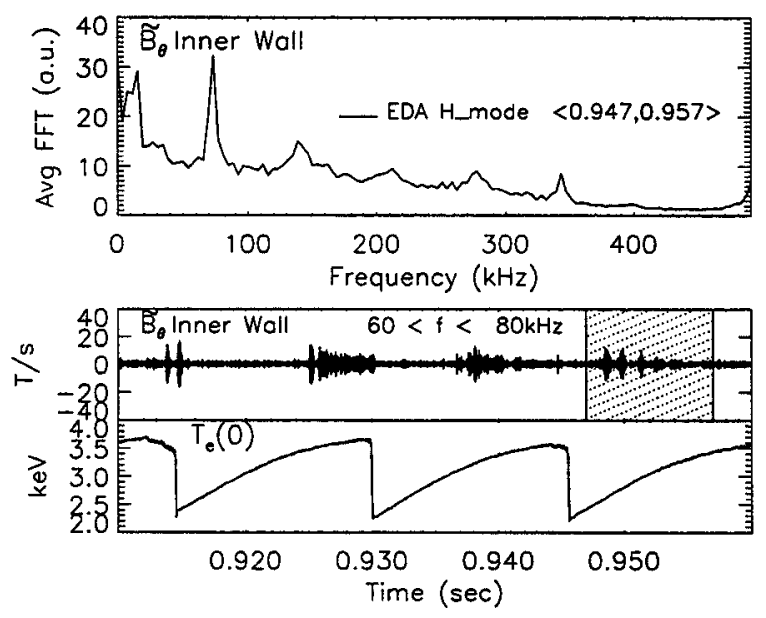

Figure 1. Fast Fourier transform of an inner wall poloidal field pick-up coil signal during an EDA H-mode averaged from 0.947 to $0.957 \mathrm{~s}$. The time history of the signal has been filtered from 60 to $80 \mathrm{kHz}$ to enhance the coherent fast edge modes. Comparison with a central electron temperature signal shows that the magnetic fluctuations occur at all phases of the sawtooth oscillations.

\section{Fluctuations during Enhanced $\mathrm{D}_{\alpha} \mathrm{H}$-mode}

The fluctuations present in Enhanced $\mathrm{D}_{\alpha} \mathrm{H}$-mode do not exhibit the characteristics of previously defined ELM types. There are bursting or continuous coherent fast edge magnetic oscillations with frequencies from 60 to $90 \mathrm{kHz}$ that have low poloidal and toroidal mode numbers, typically $\mathrm{m}=4, \mathrm{n}=1$. There are also continuous density fluctuations with peak frequencies from 50 to $200 \mathrm{kHz}$ as well as broadband magnetic and density fluctuations out to $300-400 \mathrm{kHz}$. The coherent magnetic fluctuations exhibit no ballooning character in that, once the signals are corrected for the distance to the mode rational surface, the outboard limiter signals are about the same 
amplitude as the inner wall signals $\left(\tilde{B}_{\theta} / \mathrm{B}_{\theta} \sim 5 \times 10^{-5}\right)$. The mode rational surface is calculated to be only $4 \mathrm{~mm}$ inside the last closed flux surface. The density and magnetic fluctuations may appear together or separately with nearly the same or with quite different frequencies, but no clear coherence has been found between the magnetic and density fluctuations even though they appear to originate from the same edge region within the plasma.

Figure 1 shows an example of fast edge modes measured with a poloidal field pickup coil at the inner wall during an EDA H-mode together with a Fourier spectrum of the raw signal. The time trace of the signal has been filtered from 60 to $80 \mathrm{kHz}$ to enhance the features of the mode. A central electron temperature signal is also shown to compare the timing of the fast edge mode bursts with the sawtooth crashes. The bursts occur independent of the phase of the sawtooth. The Fourier spectrum shows several harmonics of the $\sim 70 \mathrm{kHz}$ fast edge mode, which could be higher order harmonics of the dominant $\mathrm{m}=4, \mathrm{n}=1$ mode. Note that the EDA H-mode often occurs with no evidence of coherent fast edge modes, though there is generally an increase in the high frequency broadband density and magnetic fluctuations.
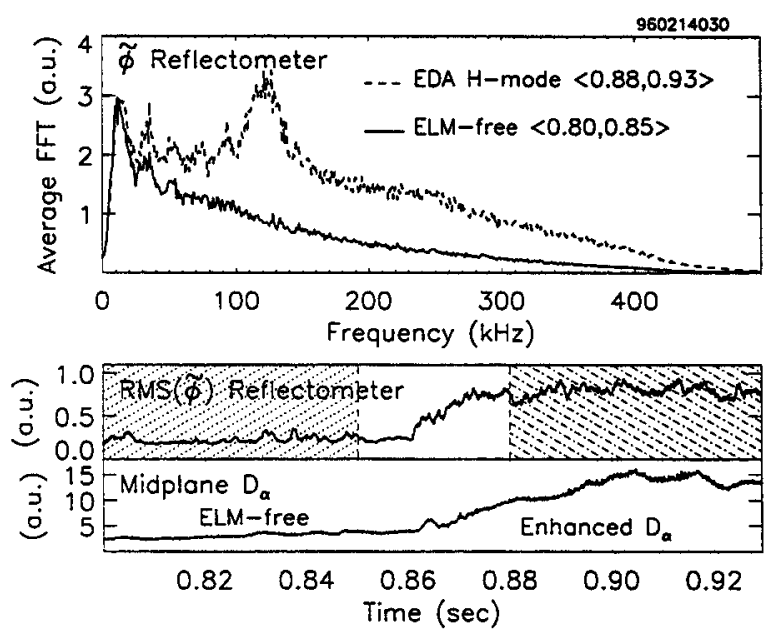

Figure 2. Comparison of fast Fourier transforms of an edge reflectometer signal averaged from 0.88 to $0.93 \mathrm{~s}$ during an EDA $\mathrm{H}$-mode (dashed) and averaged from 0.8 to $0.85 \mathrm{~s}$ during a preceding ELM-free H-mode (solid) showing an increase in broadband fluctuations out to at least $400 \mathrm{kHz}$ as well as a clear peak around $120 \mathrm{kHz}$ during EDA H-mode. The time histories of the root mean square density fluctuations and the $\mathrm{D}_{\alpha}$ emission show that the fluctuations increase when the $\mathrm{D}_{\alpha}$ emission begins to increase and remain higher throughout the EDA H-mode.

In addition to the magnetic fluctuations, the high frequency broadband density 
fluctuations, observed on the edge reflectometer signal, increase markedly during an EDA H-mode. Figure 2 shows a clear example of an ELM-free $\mathrm{H}$-mode becoming an Enhanced $\mathrm{D}_{\alpha} \mathrm{H}$-mode at about $0.86 \mathrm{sec}$ as the $\mathrm{D}_{\alpha}$ emission begins to increase. At the same time, the broadband density phase fluctuations on the reflectometer signal increase out to at least $400 \mathrm{kHz}$ and a broad peak in the spectrum appears around $120 \mathrm{kHz}$. The RMS of the reflectometer phase fluctuations increases coincident with the increase in the $D_{\alpha}$ emission and remains higher throughout the EDA phase. The magnetic fluctuations during the EDA phase of this discharge do not show any clear peaks in the spectrum.

\section{Comparison with Theory}

Since fast edge modes appear to be quite localized in the steep gradient region at the edge in $\mathrm{H}$-mode, it is plausible that temperature, density, or pressure gradients could be involved in destabilizing these modes. The coherent magnetic fluctuations have frequencies that are many times the estimated diamagnetic drift frequency, $\omega_{* e}$, suggesting that a strong edge rotation may be present, which is in the opposite direction to the measured core rotation [12].

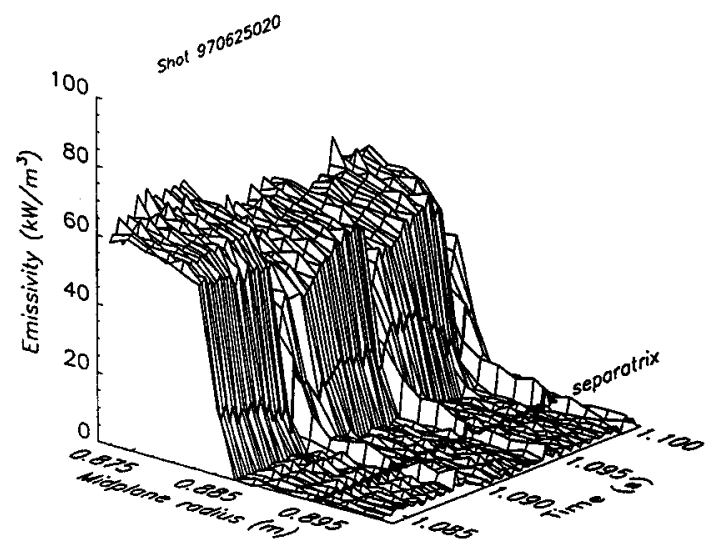

Figure 3. Profile of the reconstructed edge soft $\mathrm{x}$ ray emissivity versus time showing a series of three Type III ELMs. A steep edge pedestal exists between ELMs that extends over only two channels each separated by $1.7 \mathrm{~mm}$ radially. The radii are mapped to the outboard midplane assuming constant emission on a flux surface.

Initial estimates from edge electron temperature measurements gave pedestal widths of about $1 \mathrm{~cm}$ in $\mathrm{H}$-mode. Recent edge soft $\mathrm{x}$ ray measurements indicate 
that a combination of the edge temperature and density gradients is much steeper with pedestal widths of about 2 to $4 \mathrm{~mm}$ (Figure 3 ). The steep gradient region in the reconstructed emission between Type III ELMs occurs over only two spatial channels separated by about $1.7 \mathrm{~mm}$ when mapped back to the midplane assuming constant emission on a flux surface. The pedestal collapses within about $100 \mu \mathrm{sec}$ during each ELM. Assuming similar temperature and density pedestals, initial calculations indicate that the soft $x$ ray pedestal width is about $70 \%$ of the temperature or density width.

Preliminary calculations of ideal and resistive modes have been performed with the BALOO code [13], the CAXE and KINX codes [14], and the MARS code [15]. C-Mod equilibria in which fast edge modes are observed, with edge pressure pedestal widths of $7 \mathrm{~mm}$, are found to be stable to ideal ballooning modes, though the edge pressure gradients are within $15-30 \%$ of being unstable. If the pressure gradients are as steep as the soft $\mathrm{x}$ ray gradients, then ideal or resistive ballooning modes may be unstable.

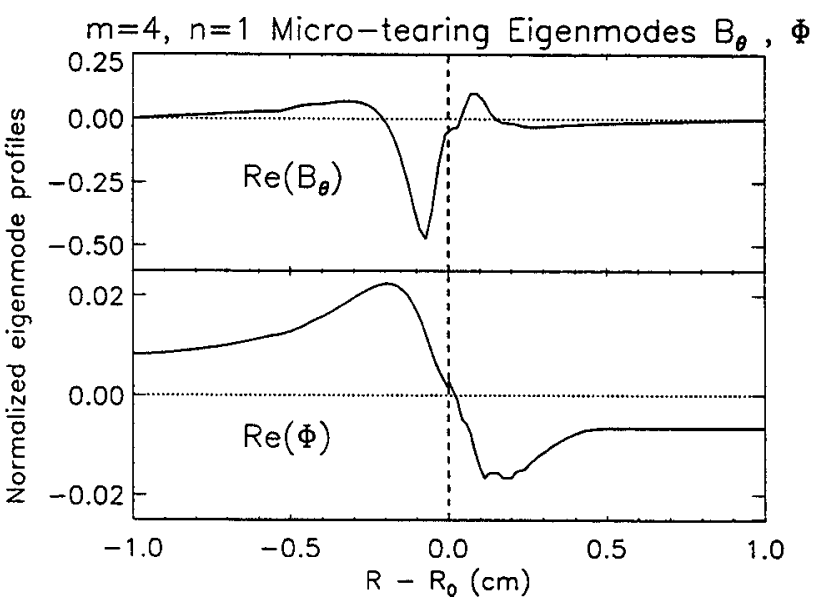

Figure 4. Normalized $m=4, n=1$ micro-tearing eigenmodes of the poloidal field, $\mathrm{B}_{\theta}$, and the electrostatic potential, $\Phi$,showing their localization to the mode rational surface $R_{0}$.

Other possible modes whose stability has been examined are the edge micro-tearing modes $[9,10]$. Their eigenmodes are localized to a few ion gyroradii $\left(\rho_{i} \sim 1 \mathrm{~mm}\right)$ of the rational surface (Figure 4 ) and are driven by steep temperature gradients. Microtearing modes are predominantly electromagnetic but have a smaller electrostatic component and they are not ballooning. The eigenmode solver from Ref. [10], which 
assumes concentric, circular flux surfaces in a large aspect ratio limit, has been used to examine the stability of $\mathrm{m}=4, \mathrm{n}=1$ radial eigenmodes in a plasma characterized by $\mathrm{L}_{n} \sim \mathrm{L}_{T} \sim 0.5 \mathrm{~cm}$ and $\beta \sim 0.002$ and $\mathrm{n}_{e}=2.7 \times 10^{20} \mathrm{~m}^{-3} T_{e}=T_{i}=300 \mathrm{eV}$ at the top of the pedestal. The fundamental $\mathrm{m} / \mathrm{n}=4 / 1$ radial eigenmode displays the largest growth rate. Unstable higher radial harmonics and higher $m$ modes also appear to be present. The higher radial harmonics of the $m=4, n=1$ eigenmodes and the higher $m$ modes may only be detectable by internal density fluctuation measurements because of the rapid decay with distance from the rational surface.

Since the coherent fast edge modes are nearly in-out symmetric and have low $\mathrm{n}=1$, it seems unlikely that they could be related to ballooning modes. The high frequency broadband turbulence may be unresolved multiple high $\mathrm{m}, \mathrm{n}$ modes and more calculations still need to be done to make detailed comparisons of resistive ballooning, micro-tearing, and other modes with the experiment.

\section{References}

[1] Wagner F, etal 1982 Phys. Rev. Lett 491408

[2] Doyle E J, Burrell K H, Lehecka T, Luhmann N C Jr, Matsumoto H, Osborne T Peebles W A, Philipona R and Rettig C 1991 Proc. 18th EPS Conf. on Controlled Fusion and Plasma Physics (Berlin) 15C Part I p 285

[3] Hutchinson I H, etal 1994 Phys. Plasmas 11511

[4] Ryter F, H-mode Database Working Group, 1996 Nucl. Fusion 361217

[5] Takase Y, etal 1997 Phys. Plasmas 41647

[6] Greenwald M, etal 1997 Nucl. Fusion 37793

[7] Snipes J A, etal 1997 Proc. 24th EPS Conf. on Controlled Fusion and Plasma Physics (Berchtesgaden) to be published

[8] Hutchinson I H, Granetz R S, Hubbard A, Snipes J A and Wolfe S M, 1997 Proc. 24th EPS Conf. on Controlled Fusion and Plasma Physics (Berchtesgaden) to be published

[9] Gladd N, Drake J, Chang C, Liu C S, 1980 Nucl. Fusion 231182

[10] Lau Y T, 1990 Nucl. Fusion 30965

[11] Stek P C, Reflectometry Measurements on Alcator C-Mod PhD thesis, MIT 1997

[12] Rice $J$ E, etal, "Observations of Central Toroidal Rotation in ICRF Heated Alcator C-Mod Plasmas", this conference

[13] Miller R L, Lin-Liu Y R, Osborne T H, and Taylor T S, "Ballooning mode stability for selfconsistent pressure and current profiles at the H-mode edge", this conference

[14] Medvedev S, etal 1993 Proc. 20th EPS Conf. on Controlled Fusion and Plasma Physics (Lisbon) 17C Part IV 1279

[15] Bondeson A, Vlad G, Lütjens H, 1992 Phys. Fluids 41889 